\title{
Evaluación De La Eficacia Del Implante Coclear En Función De La Edad De Implantación
}

\author{
Alegre, $0 . M$ \\ Rodríguez, M. C
}

Departamento de Didáctica e Investigación Educativa. Universidad de La Laguna

Villar, L. M

Departamento de Didáctica y Organización Escolar. Universidad de Sevilla Pérez, $D$

Departamento de Didáctica e Investigación Educativa. Universidad de La Laguna

doi: 10.19044/esj.2016.v12n26p42 URL:http://dx.doi.org/10.19044/esj.2016.v12n26p42

\begin{abstract}
Introduction and objective: Childhood hearing loss affects cognitive, emotional and language development of children causing difficulties in communication. Therefore, many organizations stress the importance of early detection of hearing loss and early cochlear implantation. The purpose was to verify the effect of cochlear implants performed in children from Tenerife (sorted by chronological age, age at cochlear implantation and time of use of cochlear implant) in lexical comprehension and communication skills.

Method: A psycholinguistic profile of all children implanted in the province of Santa Cruz de Tenerife during the years 2007-2012 using two standardized tests were developed: The Illinois Test of Psycholinguistic Abilities (ITPA) and the Picture Vocabulary Test (Peabody).

Results: Most of the children studied were implanted at a late age (over 3-yearolds). This situation carries some functional use of the implant but causes an indiscernible development of oral communicative functions of children. Specifically, $20 \%$ of the sample ( 28 children) was implanted with less than 2year-olds; another $28 \%$ of children was implemented between the ages of 2 and 3 years old; finally, 58\% of children was implemented with more than 3year-olds. The latter age of implantation may affect the standard language development.
\end{abstract}

Conclusions: This study has become evident that early implantation has a positive effect on language development of deaf children. 
Keywords: Cochlear implant; language development; communicative function

\section{Resumen}

Introducción y objetivo: La hipoacusia infantil repercute en el desarrollo cognitivo, emocional y lingüístico de niñas y niños ocasionando dificultades en su comunicación. Por ello, muchos organismos destacan la relevancia de la detección temprana de la hipoacusia y la implantación coclear precoz. El propósito fue constatar el efecto de los implantes cocleares realizados en las niñas y los niños de Tenerife (clasificados por edad cronológica, edad de implantación coclear y tiempo de uso del implante coclear) en la comprensión léxica y en la capacidad comunicativa.

Método: Se elaboró un perfil psicolingüístico del total de niñas y niños implantados en la provincia de Santa Cruz de Tenerife durante los años 2007 a 2012 mediante dos pruebas estandarizadas: el Illinois Test of Psycholinguistic Abilities (ITPA) y el Test de Vocabulario en Imágenes (Peabody).

Resultados: La mayoría de los casos estudiados fueron implantados (con más de 3 años de edad). Esta situación conllevó un desarrollo imperceptible de las funciones comunicativas orales de niñas y niños. En concreto, el $20 \%$ de la muestra (28 casos de niñas y niños) fue implantado con menos de dos años de edad; otro $28 \%$ de casos fue implantado entre los dos y tres años; finalmente, el 58\% de las niñas y los niños fue implantado con más de tres años. Esta última edad de implantación puede afectar el desarrollo normalizado del lenguaje de una niña o niño.

Conclusiones: Este estudio ha hecho patente que la implantación temprana tiene un efecto positivo sobre el desarrollo del lenguaje de los niños y niñas sordos.

Palabras clave: implante coclear; desarrollo del lenguaje; función comunicativa

\section{Introduction}

Es perentoria la necesidad de contar con profesionales que atiendan la hipoacusia en edades tempranas de niñas y niños, y que tengan experiencia en la educación de los trastornos de la audición, el habla y el lenguaje en los más pequeños. Considerada la hipoacusia neonatal un problema de salud pública, la detección precoz de la misma mediante cribado constituye en sí misma un programa de actuación que está consiguiendo una meritoria eficiencia en algunos centros hospitalarios, y en particular en el Complejo Universitario Insular Materno Infantil de Las Palmas de Gran Canaria (Boroski y cols., 2013). 
Desde todos los sectores sociales se argumenta la conveniencia de la detección precoz de la hipoacusia neonatal por cuanto las niñas y los niños pueden llegar a tener trastornos en el reconocimiento fonológico y en las destrezas léxicas para la adquisición del lenguaje (Dillon y cols., 2013).

Aunque la sordera de las personas no quiebra su conocimiento del mundo exterior, sin embargo los trastornos auditivos tienen un impacto socioeconómico y afectivo en los individuos. Por ello, se utilizan dispositivos quirúrgicamente instalados en el cráneo de las personas que tienen pérdida auditiva neurosensorial o lesiones o infecciones en el oído interno: los implantes cocleares (IC) son prótesis que ayudan a ese tipo de individuos a entender el lenguaje hablado, a percibir sonidos medioambientales o a escuchar música.

Las infecciones en las alumnas y los alumnos con IC, como la mastoiditis aguda, y los efectos adversos en el desarrollo motor grueso añaden riesgos en esos pacientes (Rodríguez y cols., 2010; De Kegel y cols., 2014). Así, se han promovido y ensayado estrategias de entrenamiento audiovisual para la rehabilitación auditiva de las niñas y los niños pequeños porque un conocimiento recíproco de la eficacia de los tratamientos - sean o no audiovisuales - entre los profesionales sanitarios y educativos y las familias para el cuidado de las niñas y los niños con pérdida de audición es ineludible e incontrovertible.

Los estudios sanitarios, en colaboración con la indagación educativa, han emprendido líneas de trabajo como la percepción auditiva de las niñas y los niños pequeños con IC y su repercusión en la audición musical. Bajo esta perspectiva se ha comparado la programación frecuencial (una es la versión software "Custom Sound Suite") con un programa estándar, dando como resultado que aquel software mejoraba la percepción y el reconocimiento de patrones musicales, escalas tonales, armónicas e instrumentos (Falcon y cols., 2014), y con mayor frecuencia se ha indagado la adquisición del lenguaje analizando, por ejemplo, las emisiones otoacústicas transitorias a través de la cóclea (Aguilera y cols., 2015).

El conocimiento del vocabulario en poblaciones de alumnas y alumnos sordos es menor que el de sus compañeros, así como su rendimiento académico a nivel universitario. Parece que se atribuye esa diferencia a no tener acceso a conversaciones con otros compañeros de audición considerada normal. Asimismo, se ha constatado que las niñas y los niños con pérdida de audición tienen problemas de conducta que además aumentan cuando tienen menos capacidad de lenguaje (Stevenson y cols., 2010). De otra parte, el uso de signos para la ampliación de la comunicación hablada influye negativamente en la velocidad del ensayo verbal y esta a su vez en posteriores resultados. Así, pues, se ha concluido que el modo de comunicación temprana 
ejerce una poderosa influencia en los primeros resultados que persisten en los años posteriores (Geers y cols., 2010).

Estudios de seguimiento individualizados en ambientes familiares de niñas y niños sordos con casos de IC unilateral mediados por las actitudes positivas de los padres hacia interacciones significativas y la animación de la actividad bimodal, como el caso en el que se combinaba el lenguaje de signos y la palabra hablada, puede ayudar a construir en los sujetos una segunda lengua (Cramér, 2013). El ambiente familiar de las niñas y los niños con IC se puede modificar por medio de terapias o programas educativos hasta alcanzar suficiente robustez en la creación de ambientes de aprendizaje del lenguaje.

La educación inclusiva de las niñas y los niños con IC representa consecuentemente un terreno de desafíos curriculares y de gestión en la comunicación para el profesorado así como dificultades entre los propios escolares para mantener una interacción productiva. Los programas e intervenciones educativas para la interacción social son prometedores cuando la plantilla docente o los profesores itinerantes han recibido una formación inicial en los grados universitarios que los capaciten para colaborar con otros profesionales, proporcionar enseñanza directa, recoger y analizar información, y ser miembros eficaces de equipos de trabajo, cuestiones que resultan así resumidas en sondeos de opinión entre maestros itinerantes (Luckner y Ayantoye, 2013). Como se ha constatado en un estudio, cuando las niñas y los niños con IC de Educación Infantil cursan un programa de entrenamiento de aprendizaje rápido de palabras éstos desarrollan un conocimiento léxico receptivo por capas que indica su maleabilidad (o número de palabras aprendidas rápidamente y que pueden aumentar con la práctica) (Lund y Schuele, 2014).

El objetivo del presente estudio fue constatar el efecto de los implantes cocleares realizados en las niñas y los niños en la isla de Tenerife en la comprensión léxica y en la capacidad comunicativa.

\section{Método:}

\section{Sujetos}

La muestra de participantes estuvo compuesta por 28 del total de la población de 30 niñas y niños con IC en edades comprendidas entre los 2 y los 14 años de la Isla de Tenerife, siendo la edad media de ocho años. Todas las niñas y los niños participantes se hallaban distribuidos en siete centros educativos de los 31 municipios de la Isla de Tenerife.

\section{Instrumentos}

Se administraron dos tests estandarizados a las niñas y los niños participantes. Las pruebas estandarizadas tuvieron como meta evaluar las siguientes acciones: (a) comprensión léxica mediante el Test de Vocabulario 
en Imágenes (Peabody) (Dunn y cols., 1986) y (b) comprobar las habilidades lingüísticas de las niñas y los niños en sus procesos de comunicación y fijar su nivel de adquisición del lenguaje (percepción, interpretación y transmisión) por medio del Test Illinois de Habilidades Psicolingüísticas (ITPA) (Kira y cols., 1996).

\section{Diseño y procedimiento}

Se estableció una reunión con los profesionales de los gabinetes de logopedia que habían intervenido con las niñas y los niños implantados antes de la aplicación de los dos tests. Posteriormente, se les entrenó, en los casos necesarios, en la administración de las pruebas Peabody e ITPA. En el caso del test Peabody, su aplicación a las niñas y los niños fue de forma individual en una sola sesión de 10 a 20 minutos de duración. La administración estándar del test ITPA se condujo de forma individual y en cuatro sesiones de 45 minutos. Transcurridas dos semanas, un investigador del equipo se volvió a reunir con los logopedas para recoger las pruebas y dos investigadores analizaron las puntuaciones estandarizadas obtenidas.

\section{Análisis de datos}

Se exploró la posible relación entre los perfiles psicolingüísticos de las niñas y los niños implantados y sus correspondientes edades. El análisis de datos se realizó utilizando como variables la Edad Cronológica (E.C., niñas y niños con menos de 2 años, casos entre 2 y 4 años, entre 5 y 10 años y más de 10 años), la Edad Equivalente (E.EQ., presupone que las puntuaciones en el vocabulario auditivo se distribuye normalmente), la edad de implantación coclear (casos con menos de 2 años, niñas y niños entre 2 y 4 años, y con más de 4 años) y el tiempo de uso del implante coclear (niñas y niños con menos de 3 años y con más de 3 años).

La clasificación demográfica de los sujetos por factores (edad cronológica, edad de implantación coclear, edad de rehabilitación de la sordera, escucha normal, etc.) se ha tenido en cuenta a fin de comprobar la relación entre la puntuación de un test y un factor temporal, como la Edad Auditiva (E.A.) (tiempo transcurrido desde la primera programación del IC hasta el momento de la evaluación), según el ITPA (Sarant y cols., 2008).

\section{Resultados:}

\section{Análisis descriptivo de las pruebas}

La medición de la comprensión auditiva y el desarrollo del lenguaje oral, así como del vocabulario de las niñas y los niños con IC, reflejó que un $22 \%$ de los estudiantes implantados tempranamente mostraron una comprensión sensible del lenguaje y una adecuación de vocabulario. Sin embargo, los escolares implantados a edades tardías mostraron resultados 
bajos en el conocimiento del vocabulario receptivo, según la prueba Peabody. La Figura 1 representa a los tres grupos (niños y niñas con IC antes de los 2 años, entre los 2-3 años y después de los 3 años) y la comparativa de la E.C. (línea azul) con la edad equivalente auditiva (E.EQ., línea roja) del test de vocabulario de dibujos Peabody.

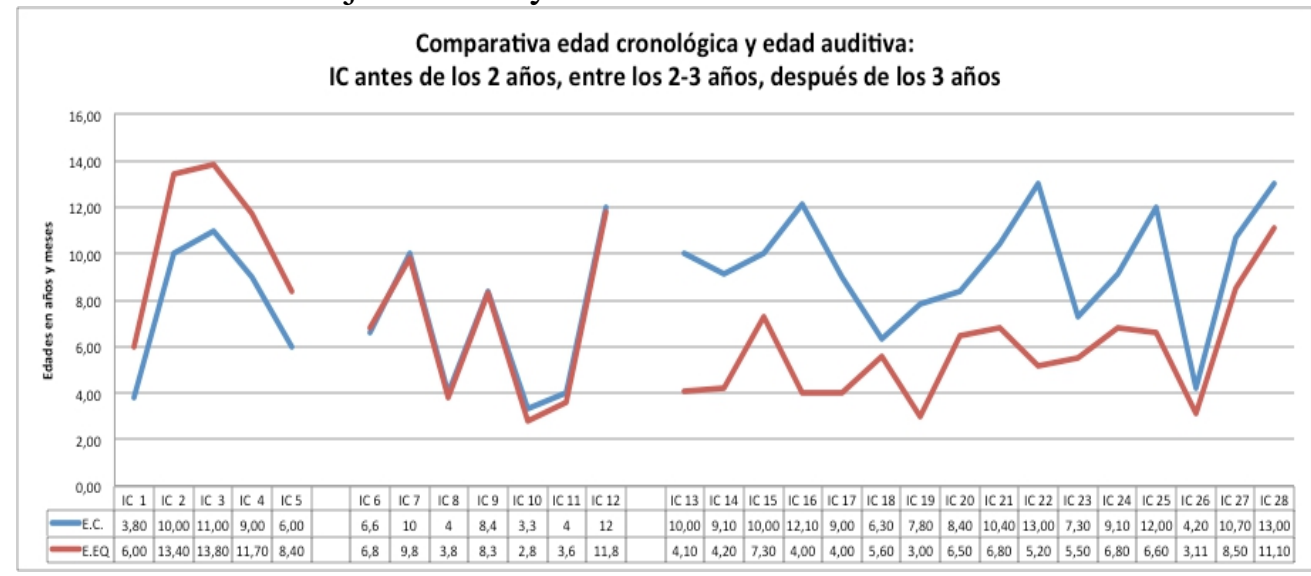

Figura 1. Comparativa de la E.C. y la E.EQ. equivalente en niñas y niños con IC llevado a cabo antes de los 2 años, entre los 2-3 años y después de los 3 años, según la prueba Peabody.

Se observó en los 5 niños con IC realizado antes de los 2 años, que en todos los casos la comparación entre la E.C. y la E.EQ., fue superior en el segundo caso. Así, la niña o el niño de 3,8 años (IC 1) poseía una E.A. de 6 años y el de 6 años (IC 5) era de 8,4 años. En los casos de los implantados IC 2, IC 3, IC 4 entre los 9 y 11 años de E.C., el incremento fue de 13,4, 13,8 y 11,7 años en la equivalente E.EQ., respectivamente.

El segundo grupo correspondió a niñas y niños con IC realizado entre los 2 y 3 años (IC 6 a IC 12). Los datos señalaron que existió una equivalencia entre ambas edades, es decir, que el efecto del implante desde el punto de vista de la E.EQ. tendió a ser más baja que la E.C., excepto en IC 6.

El tercer grupo describió a niñas y niños cuyo IC se hizo después de los 3 años (IC 13 a IC 29). Claramente los datos manifestaron que su efecto fue mucho menor en la paridad de E.EQ. y E.C., siendo ésta más alta en todos los escolares. Obsérvese, no obstante, el caso IC 27 que tenía una E.C. pequeña $(4,20)$, que obtuvo una puntuación de E.EQ. $(3,11)$ y que apuntaba hacia una consonancia en el vocabulario receptivo.

Las Figuras 2 y 3 representan el perfil psicolingüístico de cada niña y niño, según la edad cronológica y la edad auditiva (E.C. y E.A respectivamente). 
ITPA: Perfil psicolingüístico (P.P1) considerando la edad cronológica (E.C.)

"E.C. $=P . P 1$

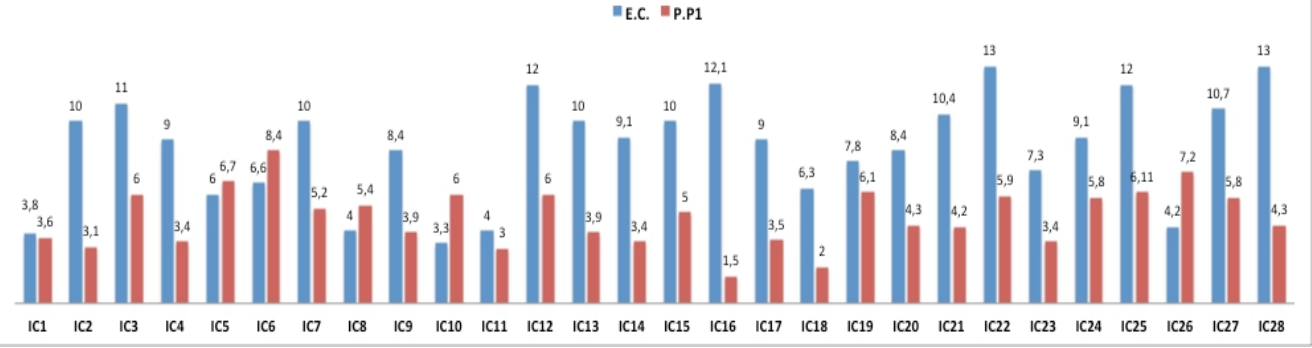

Figura 2. Perfil psicolingüístico de las niñas y los niños con IC según resultados obtenidos en el ITPA para la edad cronológica (E.C.).

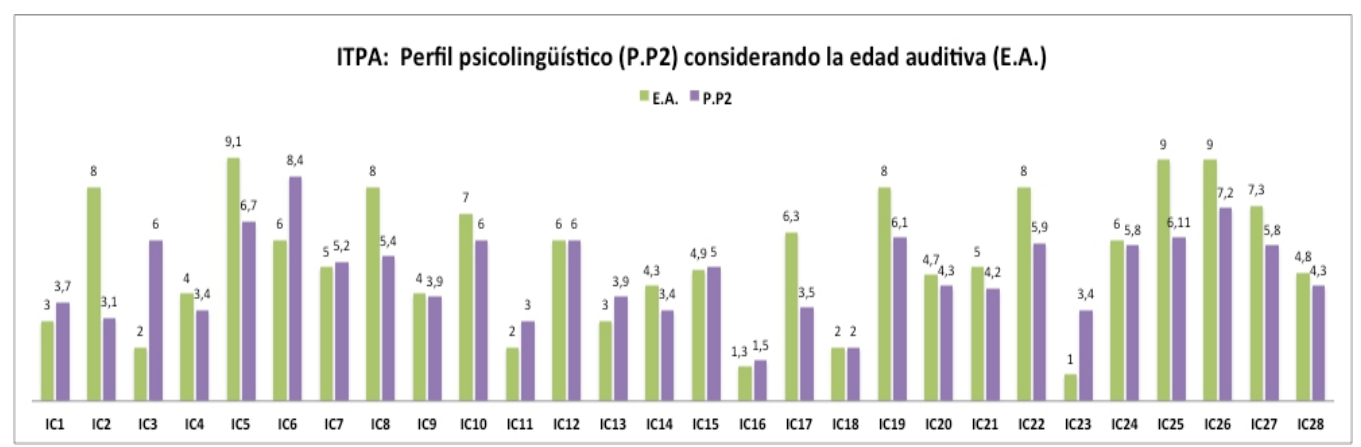

Figura 3. Perfil psicolingüístico de las niñas y los niños con IC según resultados obtenidos en el ITPA para la edad auditiva (E.A.).

Como se puede observar en las dos figuras anteriores, en el caso de la Figura 3 está más cercano el perfil psicolingüístico a la edad auditiva, mientras que en la Figura 2 que refleja el perfil psicolingüístico en función de la edad cronológica manifiesta una distancia mayor. Es decir, a la hora de estudiar el perfil psicolingüístico de las niñas y niños con IC resulta más oportuno y realista utilizar la E.A. que la E.C.

En relación con los factores del ITPA, ocurrió una prevalencia de los procesos psicolingüísticos del canal visual (por ejemplo, la comprensión visual, integración visual, asociación visual, la expresión motora y el factor memoria secuencial visomotora en el caso del IC 12) sobre los auditivos (excepción hecha de IC 1, donde los factores auditivos, por ejemplo, comprensión auditiva, asociación auditiva, memoria secuencial auditiva, integración auditiva, expresión verbal e integración gramatical, fueron superiores a los visuales). Asimismo, se registró una tendencia en el predominio de los procesos psicolingüísticos de carácter visual en niñas y niños con IC tardío y una propensión al uso de procesos de carácter auditivo en edades tempranas de IC. Se suponía que tras un IC la fuerza de los procesos receptivo y asociativo de carácter visual sería menor, pero se advirtió que no sucedía de esta manera en los casos observados. Según la E.A. de las niñas y 
los niños, se subraya que los resultados mejoraron con respecto a la E.C. lo que reveló que el IC fue un dispositivo compensador de las funciones comunicativas y del lenguaje afectadas por la sordera.

En función de la E.C., resultó que las niñas y los niños de más de 3 años revelaron una predisposición a la disminución de las puntuaciones en los procesos receptivo y asociativo de naturaleza auditiva, y los de menos de 2 años manifestaron una tendencia al aumento de las puntuaciones referidas a los procesos psicolingüísticos visuales. En cuanto a la E.A., se indica que cuando la niña o el niño es implantado tempranamente, más bajo puntúa en los procesos receptivo y asociativo visuales, observándose una disposición a la mejora de los procesos auditivo receptivo y asociativo. Respecto al resultado de la implantación tardía se reparó que las niñas y los niños registraron calificaciones más bajas en los factores auditivos receptivo y asociativo y mejoraron de forma progresiva sus puntuaciones en los procesos visuales receptivo y asociativo.

\section{Discusión}

Este estudio demostró que el IC en las niñas y niños representó un beneficio sustancial en sus perfiles psicolingüísticos, es decir, obtuvieron un rendimiento positivo en las destrezas del lenguaje, como se había producido análogamente en otro estudio (Holman y cols., 2013). En efecto, la IC temprana produce ventajas en el rendimiento del vocabulario receptivo, con lo cual este estudio se alinea con otros que han demostrado una relación directa entre la edad de implantación y los resultados de lenguaje hablado (Nicholas y Geers, 2013).

Cuando se ha usado el test Peabody, se ha podido constatar el progreso realizado en el vocabulario receptivo tras la IC en niñas y niños con menos de 2 años, cuyas ratios concuerdan con los hallados en otro estudio (Hayes y cols., 2009).

Asimismo, constatamos la dificultad metodológica que tienen los estudios que aplican pruebas diagnósticas similares a las de este estudio para trabajar con niñas y niños muy pequeños.

\section{Conclusion}

Un IC realizado en niñas y niños antes de los 2 años representó una edad de lenguaje equivalente superior a la cronológica. El vocabulario receptivo de las niñas y niños con IC entre los 2 y 3 años no representó una brecha profunda entre ambas edades, si bien la E.EQ. tendió a ser más baja que la EC. Un IC realizado después de los 3 años no refleja dichos resultados. De otra parte, si tenemos en cuenta el perfil psicolingüístico obtenido a partir de la EC y la EA, muestra que en el segundo caso es más acorde a la realidad del IC. Este estudio ha hecho patente que la implantación temprana tiene un 
efecto positivo sobre el desarrollo del lenguaje de los niños y niñas sordos. Es cierto que existen múltiples factores que es preciso considerar, pero en nuestro estudio hemos evidenciado que la edad de implantación temprana es un buen predictor del adecuado desarrollo lingüístico futuro.

\section{References:}

Aguilera, S.; Gutiérrez, I.; Chamlati, L.; Alatorre, E. y Durand, A. (2015). Emisiones otoacústicas como analizador de la función coclear en niños con problemas de lenguaje. Acta Otorrinolaringológica, 66, 87-91.

Borkoski, S., Falcón, J. Bueno, J. Pérez, J., López, Z. y Ramos, A. (2013). Resultados de un programa de detección precoz de la hipoacusia neonatal (2013). Acta Otorrinolaringolologica, 64 (92-6).

Cramér, E. (2013). Sequential Bimodal Bilingual Acquisition: Mediation Using a Cochlear Implant as a Tool. Deafness \& Education International, 15, 201-21.

De Kegel, A.; Maes, L.; Van Waelvelde, H. \& Dhooge, I. (2014). Examining the Impact of Cochlear Implantation on the Early Gross Motor Development of Children With a Hearing Loss. Ear \& Hearing. doi: 10.1097/AUD.0000000000000133.

Dillon, C.M., De Jong, K. \& Pisoni, D.B. (2012). Phonological Awareness, Reading Skills, and Vocabulary Knowledge in Children Who Use Cochlear Implants. Journal of Deaf Studies and Deaf Education, 17, 205-26.

Dunn. L.; Padilla, E.; Lugo, D. \& Dunn, L. (1986). Test de vocabulario en imagines. (Peabody). Madrid: TEA.

Geers, A.E.; Strube, M.J.; Tobey, E.A,; Pisoni, D.B. \& Moog, J.S. (2010). Epilogue: Factors Contributing to Long-Term Outcomes of Cochlear Implantation in Early Childhood. Ear \& Hearing, 32, 84S-92S.

Falcón, J.C.; Borkoski, S.; Limiñana, J.M. y Ramos, A. (2014). Reconocimiento auditivo musical y melódico en pacientes con implante coclear, mediante nuevo método de programación de asignación frecuencial. Acta Otorrinolaringologica, 65, 289-96.

Hayes, H.; Geers, A.E.; Treiman, R. \& Moog, J.S. (2009). Receptive Vocabulary Development in Deaf Children with Cochlear Implants: Achievement in an Intensive Auditory-Oral Educational Setting. Ear \& Hearing, 30, 128-35.

Holman, M.A.; Carlson, M.L.; Driscoll, C.L.; Grim, K.J.; Petersson, R.S. \& Sladen D.P. (2013). Flick RP Cochlear implantation in children 12 months of age and younger. Otology \& Neurotology, 34, 251-258.

Kira, S.A; McCarthy, J.J. \& Kira, W.D. (1996). Test Illinois de Aptitudes Psicolingüisticas (ITPA). Madrid: TEA. 
Luckner, J.L. \& Ayantoye, C. (2013). Itinerant Teachers of Students Who Are Deaf or Hard of Hearing: Practices and Preparation. Journal of Deaf Studies and Deaf Education, 18, 409-23.

Lund, E. \& Schuele, C.M. (2014). Effects of a Word-Learning Training on Children With Cochlear Implants. Journal of Deaf Studies and Deaf Education, 19, 68-84.

Nicholas, J.G. \& Geers, A.E. (2013). Spoken Language Benefits of Extending Cochlear Implant Candidacy Below 12 Months of Age. Otology \& Neurotology, 34, 532-538.

Rodríguez, V.; Cavallé, L.; De Paula, C. y Morera, C. (2010). Tratamiento de la mastoiditis aguda en niños con implante coclear. Acta Otorrinolaringológica, 61,180-3.

Sarant, J.Z.; Holt, C.M.; Dowell, R.C. \&Rickards, F.W. (2008). Spoken Language Development in Oral Preschool Children With Permanent Childhood Deafness. Journal of Deaf Studies and Deaf Education, 14, 20517.

Stevenson, J.; McCann, D.; Watkin, P.; Worsfold, S. \& Kennedy, C. (2010). On behalf of the Hearing Outcomes Study Team. The relationship between language development and behaviour problems in children with hearing loss. Journal of Child Psychology and Psychiatry, 51, 77-83. 